\title{
Distribution of polymorphic marker of genes of the renin-angiotensin system RAS (AGT, AGTR1, ACE), ITGB3, PPARG) in pa- tients with essential arterial hypertension depending on the nature of the nocturnal de- crease of BP.
}

\author{
T. Yu. Zotova ${ }^{1}$, M. M. Azova ${ }^{1}$, A. A. Lukanina ${ }^{1}$, A. Ait Aissa ${ }^{1}$, M. L. Blagonravov ${ }^{1}$, \\ ${ }^{1}$ FGAOU VO "Peoples' Friendship University of Russia", Moscow, Russia. \\ Received: November 26, 2020. Revised: May 6, 2021. Accepted: June 2, 2021. Published: June 3, 2021.
}

\begin{abstract}
A clinical-genetic study using ABPM (24-hour BP monitoring) and Holter's ECG methods in 49 patients with essential arterial hypertension (group 1: 17 patients without sufficient nocturnal $\mathrm{BP}$ decrease $\mathrm{CI} \leq 10 \%$, and group 2: 32 patients with sufficient nocturnal BP decrease $\mathrm{CI} \geq \mathbf{1 0 \%}$,) was performed for comparative analysis of the genotype frequencies of $A C E, A G T$, AGTR1, ITGB3, and PPARG. The study was conducted in order to clarify the pathogenetic mechanisms of the implementation of different dynamics of nocturnal blood pressure in patients with hypertension without metabolic syndrome. It was found that in group 1, protective genotype II of the $A C E$ gene was more common $(p \leq 0.025)$ than in the population data. A significant increase ( $p \leq$ 0.025 ) in the frequency of the $C C$ genotype of the AGTR1 gene responsible for the formation of insulin resistance compared to the population data was combined with a significant increase in the frequency of autonomic dysfunction in patients of group $1-83.4 \%$ vs. $64.5 \%$ group 2 respectively. The results obtained indicate the possible pathogenetic links between genetically determined insulin resistance and autonomic nervous system dysfunction and allows us to determine therapeutic approaches for correcting the nocturnal blood pressure profile.
\end{abstract}

Key words: ABPM, essential arterial hypertension (EAH), RAS gene polymorphism (AGT, AGTR1, ACE), ITGB3, PPARG, nocturnal decrease of BP.

\section{INTRODUCTION}

Essential arterial hypertension (EAH), being a multifactorial disease, also has a genetic component in the form of polymorphism of candidate genes implemented in single-nucleotide DNA substitutions. These substitutions, by changing the properties of proteins, provide a change in the activity of, for example, proteins of the renin-angiotensin system
(RAS) [1.2.3]. It is the increased activity of RAS proteins in carriers of polymorphic markers of RAS genes that ensures the stabilization of elevated blood pressure at hypertension and provides a pathogenetic link with tissue effects. In this regard, the study of the frequency characteristics of genotypes in the presence of polymorphic gene variants is aimed at identifying candidate genes that increase the risk of developing EAH and its specific clinical forms-occurring with and without a nocturnal decrease of BP $[4,5,6,7,8]$. The selection of polymorphic markers of the AGTR1 and PPARG genes was aimed at clarifying the role of the genetic component in the formation of nocturnal insulin resistance

The study of the diurnal chronostructure of BP in EAH led to the formation of the concept of a nocturnal BP, determined on the basis of the diurnal index (DI), according to which patients are subdivided in 4 different groups: dippers, non-dippers, over-dippers and night - pickers. According to the literature, the absence of a nocturnal decrease of BP is associated with the realization of vascular dysfunction against the background of a vegetative imbalance in the form of sympathicotonia and a decrease in the characteristic nocturnal period of increasing the tone of the parasympathetic nervous system [7]. Taking into account the pathogenetic links of increased activity of RAS proteins with activation of the sympathetic link of the nervous system, observed both at the central level (sodium-dependent mechanisms) and at the peripheral level (increased release of norepinephrine in sympathetic terminals) [8], the study of the frequency of occurrence of RAS gene polymorphisms ( $A C E, A G T$, $A G T R 1)$ in a group of patients with hypertension without nocturnal decrease in BP (group 1) and with nocturnal decrease in BP (group 2), in combination with the study of the frequency of genotypes of the gene ITGB3(whose polymorphic marker, A2, increases the risk of vascular catastrophes [9]) and the PPARG gene responsible for the formation of insulin 
resistance [5] (allele C (Pro12Pro)) in patients with metabolic syndrome.

Thus, the aim of the study was to clarify the pathogenetic mechanisms of the implementation of different dynamics of nocturnal blood pressure in patients with hypertension without metabolic syndrome.

\section{MATERIALS AND METHODS.}

In the study were included 49 patients. undergoing treatment and examination in Moscow polyclinic at the State Clinical Hospital No.13, with their written informed consent, without concomitant pathology that can change the nature of the course of EAH including metabolic syndrome. The volume of the sample is determined by the low frequency of occurrence of the isolated form of $\mathrm{AH}$ and the strictness of the selection criteria.

The study did not include patients with concomitant pathology that can change the nature of the course of arterial hypertension, patients with metabolic syndrome, ischemic heart disease.

To analyze the genotype frequencies for the studied genes, 2 groups of patients were formed: group 1 $(\mathrm{n}=17)$ patients with EAH with no nocturnal decrease in BP (non-dippers and night-pickers $100 \%, \mathrm{CI} \leq 10 \%$ ) and group $2(n=32)$ patients with EAH who had a nocturnal decrease in BP (dippers and over-dippers $100 \%, \mathrm{CI} \geq 10 \%$ ).

All patients received adequate therapy that reached the target values. The gender and age composition and clinical characteristics of the studied groups are presented in Table 1. For the analysis of hemodynamic parameters, a control group $(n=15)$ aged 25 to 69 years was included into the study. Genotyping by gene polymorphisms of angiotensin converting enzyme (ACE rs4646994, I/D), angiotensinogen (AGT rs699, M235T), angiotensin II type 1 receptor (AGTR1 rs5186, A1166C), integrin $\beta-3$ (ITGB3 rs5918, Leu33Pro), peroxisome proliferatoractivated receptor (PGARG rs1801282, Pro12Ala) was performed by a real-time polymerase chain reaction using the NPF Synthol and Litech reagent kits.

The material for the study was DNA isolated from a sample of venous blood. For comparison, we used the frequencies of the analyzed gene polymorphisms in healthy individuals in population studies $[10,11,12,4]$ conducted on the territory of the Russian Federation, taking into account the race of patients, since the frequency characteristics of the studied genotypes depend on these parameters.

All patients underwent ABPM (24-hour arterial BP monitoring) and 24-hour Holter's ECG monitoring. The data obtained were processed by using the EZDoctor program (AND, Japan) and the MT-100 program (Schiller, Switzerland). The average analyzed indicators: Office BPS, $\mathrm{mmHg}$, Office BPD, $\mathrm{mmHg}$, DayBPS, mmHg, NightBPS, mmHg, DayBPD, mmHg, NightBPD, mmHg, DayHR, b/s, NightHR, b/s, Time load BPS - \% of the time during the day with the changed values of BPS, Time load BPD - $\%$ of the time during the day with the changed values of d BPD, Area load BPS - \% of the area under the curve of daily BP with the changed values of BPS, Area load BPD-\% of the area under the curve of daily BP with the changed values of BPD are shown in Table 2.

Holter HR monitoring was performed to detect autonomic dysfunction: a qualitative analysis of the distribution form of RR intervals was performed. The normal form of distribution indicates the absence of autonomic dysfunction, skew to the

\section{III.RESULTS AND DISCUSSION.}

The data presented in Table 1 allow us to speak about the balance of both groups by gender, age, absence of metabolic syndrome and the level of reduction of the renal barrier (MAU). The patients of both groups did not differ in the duration of the disease and the nature of the therapy.

Despite the therapy, both groups differed significantly from the control group in terms of the main hemodynamic average indicators and the load index by time, as well as by area, except for the area index for BPD in group 1 (Table 2).

Table1. Clinical and laboratory average indicators $(M \pm m)$ of the analyzed groups of patients

\begin{tabular}{|c|c|c|}
\hline Indicator & Group 1 $(\mathbf{n = 1 8})$ & Group2 $(\mathbf{n = 3 1})$ \\
\hline Age, years & $60,9 \pm 4,22$ & $63,7 \pm 3,17$ \\
\hline Men, \% & 44 & 42 \\
\hline Women, \% & 56 & 58 \\
\hline BMI, kg/m ${ }^{2}$ & $26,9 \pm 0,49$ & $27,6 \pm 0,37$ \\
\hline WC, cm & $91,4 \pm 1,27$ & $90,4 \pm 1,06$ \\
\hline Duration of hypertension, years & $12,6 \pm 1,19$ & $13,3 \pm 1,27$ \\
\hline HbAlc, \% & $6 \%$ & $5,7 \%$ \\
\hline Creatinine, mmol / l & $84,44 \pm 3,73$ & $85,97 \pm 2,99$ \\
\hline Urea, mmol / 1 & $6,44 \pm 0,6$ & $5,99 \pm 0,45$ \\
\hline MAU, mg / 1 & $0,6 \pm 0,01$ & $0,5 \pm 0,002$ \\
\hline
\end{tabular}




\begin{tabular}{|c|c|c|}
\hline IRImkED/ml & $12,7 \pm 1,58$ & $15,3 \pm 1,1$ \\
\hline \multicolumn{2}{|c|}{ Antihypertensive therapy, \% number of patients } \\
\hline ACE and rec.ACE block & $88(\mathrm{n}=15)$ & $78(\mathrm{n}=25)$ \\
\hline$\beta$-block & $11(\mathrm{n}=2)$ & $13(\mathrm{n}=4)$ \\
\hline Diuretics & $59(\mathrm{n}=10)$ & $47(\mathrm{n}=15)$ \\
\hline Calcium antagonists & $24(\mathrm{n}=4)$ & $28(\mathrm{n}=9)$ \\
\hline
\end{tabular}

Where: MAU-microalbuminuria, IRI-immunoreactive insulin, ACE - angiotensin converting enzyme and receptor ACE blocker.

Table 2. Hemodynamic average indicators in the studied groups

\begin{tabular}{|c|c|c|c|}
\hline Indicator & Control $(\mathbf{n = 1 5})$ & Group 1 $(\mathbf{n = 1 8})$ & Group 2 (n=31) \\
\hline Office BPS, mmHg & $123,8 \pm 1,2$ & $155,3 \pm 1,1^{*}$ & $151,8 \pm 1,02^{*}$ \\
\hline Office BPD, mmHg & $75,6 \pm 1,74$ & $87,5 \pm 1,9^{*}$ & $86,1 \pm 1,1^{*}$ \\
\hline DayBPS, mmHg. & $121,3 \pm 1,85$ & $137,5 \pm 2,28^{*}$ & $140,7 \pm 1,4^{*}$ \\
\hline NightBPS, mmHg. & $104,6 \pm 2,05$ & $142,8 \pm 2,29^{*} \bullet$ & $81,1 \pm 1,67^{*}$ \\
\hline DayBPD, mmHg. & $76,8 \pm 2,01$ & $76,5 \pm 1,8$ & $64,1 \pm 1,87$ \\
\hline NightBPD, mmHg. & $63,6 \pm 1,01$ & $73,6 \pm 2,36^{*}$ & $72,9 \pm 1,51$ \\
\hline DayHR, b/s & $75,9 \pm 1,24$ & $73,5 \pm 1,64$ & $61,3 \pm 0,9$ \\
\hline NightHR, b/s & $64,3 \pm 3,14$ & $63,4 \pm 1,52$ & $58,2 \pm 3,48^{*}$ \\
\hline Time load BPS \% & $22,9 \pm 3,21$ & $55,7 \pm 5,43^{*}$ & $36,2 \pm 2,72^{*}$ \\
\hline Time load BPD \% & $18,4 \pm 2,78$ & & $13,3 \pm 1,18^{*}$ \\
\hline Area load BPS \% & $4,9 \pm 3,42$ & $33,6 \pm 5,6^{*}$ & $7,3 \pm 0,9^{*}$ \\
\hline Area load BPD\% & $4,7 \pm 0,08$ & $12,6 \pm 3,1^{*}$ & $5,5 \pm 1,28$ \\
\hline * $<<05$-the significance of differences between control and groups 1 and $2 ;$ & & \\
\hline
\end{tabular}

$\cdot p \leq 0.05$-the significance of the difference between groups

Table 3. Frequencies of genotypes and alleles of the AGT, AGTR1, ACE, ITGB3, PPARG genes

\begin{tabular}{|c|c|c|c|c|c|}
\hline $\begin{array}{c}\text { Genotypes, } \\
\text { Alleles }\end{array}$ & $\begin{array}{c}\text { Group } 1 \\
(n=17)\end{array}$ & $\begin{array}{c}\text { Group } 2 \\
(n=32)\end{array}$ & $\mathrm{p} \leq 0,05^{*}$ & $\begin{array}{l}\text { Population } \\
\text { frequencies }\end{array}$ & $\mathrm{p} \leq \mathbf{0 , 0 5 ^ { \times }}$ \\
\hline \multicolumn{6}{|c|}{ Gene $\boldsymbol{A G T}$ rs699 } \\
\hline$T T(M 235 M)$ & 0,18 & 0,187 & ID & $0,314(\mathrm{n}=115)^{1}$ & $p 2=0,07$ \\
\hline$T C(M 235 T)$ & 0,47 & $\mathbf{0 , 5 9 3}^{\times}$ & ID & 0,40 & $p 2=0,02$ \\
\hline$C C(T 235 T)$ & 0,35 & 0,22 & ID & 0,21 & ID \\
\hline Allele $C$ & 0,585 & 0,52 & ID & 0,41 & $p 1=0,084$ \\
\hline \multicolumn{6}{|c|}{ Gene AGTR1 rs5186 } \\
\hline$A A$ & $\mathbf{0 , 3 5} 5^{* x}$ & 0,593 & 0,045 & $0,66(\mathrm{n}=115)^{1}$ & $p 1=0,008$ \\
\hline$A C$ & $\mathbf{0 , 5 3} 3^{\times}$ & 0,407 & ID & 0,294 & $p 1=0,033$ \\
\hline$C C$ & $0,12^{*}$ & $0^{\times}$ & 0,009 & 0,046 & $p 2=0,015$ \\
\hline Allele $C$ & 0,385 & 0,204 & 0,091 & 0,193 & $p 1=0,052$ \\
\hline \multicolumn{6}{|c|}{ Gene ACE rs4646994 } \\
\hline II & $0,71^{\times *}$ & 0,32 & 0,03 & $0,261(n=117)^{2}$ & $p 1=0,001$ \\
\hline$I D$ & 0,29 & $0,25^{\times}$ & ID & 0,443 & $\begin{array}{l}p 1=0,067 \\
p 2=0,018\end{array}$ \\
\hline$D D$ & $0^{\times}$ & $0,41^{*}$ & 0,001 & 0,296 & $\begin{array}{c}p 1=0,001 \\
p 2=0,11\end{array}$ \\
\hline
\end{tabular}




\begin{tabular}{|c|c|c|c|c|c|}
\hline Allele D & $\mathbf{0 , 1 4 5}^{* \times}$ & 0,54 & 0,001 & 0,517 & $p 1=0,001$ \\
\hline \multicolumn{6}{|c|}{ Gene ITGB3 rs5918 } \\
\hline$T T$ & 0,82 & 0,72 & ID & $0,724(\mathrm{n}=858)^{3}$ & ID \\
\hline$T C$ & 0,18 & 0,25 & ID & 0,257 & ID \\
\hline CC & 0 & 0,03 & ID & 0,019 & ID \\
\hline Allele $C$ & 0,09 & 0,15 & ID & 0,15 & ID \\
\hline \multicolumn{7}{|c|}{ Gene PPARG rs1801282 } \\
\hline CC(Pro12Pro $)$ & 0,65 & 0,593 & ID & $0,69(\mathrm{n}=556)^{4}$ & ID \\
\hline CG(Pro12Ala $)$ & 0,29 & 0,343 & ID & 0,28 & ID \\
\hline GG(Ala12Ala $)$ & 0,06 & 0,064 & ID & 0,03 & ID \\
\hline Allele $G$ & 0,2 & 0,24 & ID & 0,17 & ID \\
\hline
\end{tabular}

* $\mathrm{p}<0.05$-the significance of differences in genotypes and alleles between groups 1 and 2 ;

$\times \mathrm{p} \leq 0.05$-the significance of the difference between the studied groups in comparison with the comparison with the population data 1 - [10]; 2 [11]; 3- [12]; 4 - [4]; ID - invalid difference

The analysis of the frequency characteristics of the genotypes of the studied genes we begin with the ITGB3 gene that reflects platelet-vascular dysfunction. On the basis of this gene, the platelet receptor integrin -3 is synthesized, which is responsible for the irreversible aggregation of platelets. In this study, the frequency characteristic of the minor C (A2) allele responsible for the development of vascular pathology in patients with ACS and arterial hypertension developing within the framework of the metabolic syndrome [4.9] was not increased, and in group 1 there was a tendency to increase the protective TT genotype and decrease the $\mathrm{C}$ allele of this gene.

The analysis of the AGT rs699 gene genotype frequencies, which leads to the replacement of M235T, led to the following results: in both groups, there was a decrease in the frequency of the TT genotype in comparison with the population data $(\mathrm{p} \leq 0.025)$. Thus, the increase in the frequency of the $T C$ genotype of the $A G T$ gene in comparison with the population data unites both groups within the general pathology in the form of EAH.

The analysis of changes in the frequency characteristics of the ACE gene I/D polymorphism led to the following results. For group 1 of patients with essential hypertension, there was a significant increase in genotype II and a decrease in the D allele both in the population and in the intergroup comparison. Group 2 patients showed a typical increase in the DD genotype of the ACE gene, which leads to an increase in the activity of the angiotensin protein in the blood plasma of patients with hypertension and is considered as a candidate gene that increases the frequency of EAH development [1].

Further, we analyzed the frequency of polymorphic markers of the AGTR1 rs5186 and PPARG rs 18001282 genes. The minor allele $\mathrm{C}$ of the AGTR 1 rs5186 gene and the major allele $\mathrm{C}$ (Pro12Pro) of the PPARG rs18001282 gene are considered in the literature as risk factors for the development of insulin resistance $[4,5,6]$. At the same time, the mechanisms of their implementation are different. Thus, the
PPARG rs18001282 gene, providing the synthesis of the gamma receptor activated by peroxisome proliferators, ensures the implementation of the tissue effects of RAS by an increase in the activity of proteins of this system. It should be noted that for both groups, there was no change in the frequency characteristics of the genotypes of this gene in comparison with the population data. However, the normal nocturnal decrease in BP, which develops against the background of an increase in the tone of the parasympathetic link of the innervation of the autonomic nervous system, was accompanied by the development of insulin resistance, which develops against the background of the action of contrinsular factors that provide endogenous nutrition of the body during sleep. The analysis of the AGTR1 gene genotype frequencies suggests that a significant increase in the frequency in group 1 of patients without an adequate nocturnal decrease in BP of the $A C, C C$ genotypes may be accompanied by a change in the nature of the autonomic regulation of the cardiovascular system. To confirm the presence of autonomic dysfunction, Holter HR monitoring was performed and it was found that in group 1, autonomic dysfunction was significantly more common: $83.4 \%$ versus $64.5 \%$ in group 2 patients.

Thus, our study provides the explanation of the specific mechanisms of the implementation of insulin resistance at night in patients with hypertension with the lack of adequate nocturnal blood pressure reduction, which occurs, among other things, due to the activation of the sympathetic link of the autonomic nervous system, which was observed in $64.5 \%$ of cases in group 1 versus $45.1 \%$ in the second group of patients. 


\section{CONCLUSION}

Currently EAH is considered from the standpoint of not only a multifactorial disease, but also a disease with different phenotypes. Thus, EAH and AH that develops within the framework of the metabolic syndrome are distinguished. The studied pathogenetic differences in the mechanisms of formation of these two clinical forms of the disease can be conditionally reduced to an assessment of the role of insulin resistance, including those that develop normally and at night, and the balance of local and central mechanisms for the implementation of insulin resistance, their relationship with the activity of RAS proteins.

This problem is most significant for patients with EAH in the absence of adequate BP reduction (group 1). The absence of pronounced differences in the frequency characteristics of the PPARG gene compared to the population data indicates the absence of the role of local tissue factors in the development of insulin resistance in patients with EAH of both groups. The dominance of the AC genotype of the AGTR1 gene in patients without nocturnal BP reduction (group 1), combined with the predominance of autonomic dysfunction in this group, indicates the presence of possible pathogenetic relationships of RAS proteins with autonomic regulation of HR in this group of patients. This makes it possible to predict the probability of developing an altered nocturnal BP profile and to develop therapeutic approaches for correcting insulin resistance in a specific group of patients based on the study of polymorphic markers of the AGTR 1 gene[13].

In addition, the predominance of the protective genotype II of ACE gene allows to exclude kidney damage as a cause of fixing the nocturnal increase in BP. We believe that differences in the frequencies of polymorphic markers of RAS genes may be the leading pathogenetic mechanism that provides a combina-

\section{REFERENCES}

[1] Baranov A.V., Fadeva O. Yu., Shilyaev R. R., Bedina I. V., Kuznetsova E. G. The role of polymorphism of $\mathrm{I} / \mathrm{D}$ angiotensin converting enzyme in the development of tubulointerstitial kidney lesions / / Bulletin of the Ivanovo Medical Academy. - 2012. volume 17. - No. 4. - pp. 47-50;

[2] ] Dickson M.E., Sigmund C.D. Genetic basis of Hypertension: revisiting angioten - sinogen//Hypertension. -2007.-48.-pp.14-20;

[3] Zotova T. Yu. Kubanova AG Azova M. M., Aissa A., Gigani O. O., Frolov V. A. Analysis of the polymorphism of the angiotensin system genes (ACE, AGT, AGTR1) and the ITGB3 gene in patients with arterial hypertension in combination with metabolic syndrome. - 2016. - T161, No. 3. -pp. 17-21; tion of insulin resistance with the formation of autonomic dysfunction and form the basis for the creation of an altered nocturnal BP profile.

\section{V.SUMMARY}

The results obtained in this study suggest that the polymorphic marker of the AGTR 1 gene and autonomic dysfunction are involved in the formation of an altered nocturnal blood pressure profile in group 1 patients. The role of the renal factor is unlikely due to the dominance of the genotype II of ACE gene in group 1 patients.

\section{LIST OF ABBRIVIATIONS}

EAH- essential arterial hypertension

BP - blood pressure

ACE-angiotensin converting enzyme

BPD - diastolic blood pressure

BPS-systolic blood pressure

HR - heart rate

ARBs-angiotensin II receptor blockers

BMI - body mass index

MAU-microalbuminuria

WC-waist circumference

IRI- immune-reactive insulin

АВРМ - 24-hour артериальное blood pressure monitoring

RAS-renin-angiotensin system

CI-circadian index

AGT-angiotensinogen

AGTR1-angiotensin I receptor

ITGB3-Integrin beta-3

HbA1c-glycosylated hemoglobin

PPARG-receptors activated by the peroxisome proliferator

IHD - ischemic heart disease

[4] Bondar I. A., Filipenko M. L., Shabelnikova O. Yu., Sokolova E. A. Association of the polymorphic marker rs1801282 of the PPARG PRO12ALA gene with type 2 diabetes mellitus in the Novosibirsk region and other populations//Siberian Medical Journal, 2014, vol. 29, no. 2, pp. 75-78.

[5] Kravchenko N. A., Yarmysh N. V. The role of PPARs and its isoforms in metabolic disorders associated with insulin resistance and diabetes//Cytology and genetics. - 2008. -42(4). - pp. 69-79;

[6] Kulikova M. V., Asceulova T. V. Components of the renin-angiotensin system and the role of systemic inflammation in patients with arterial hypertension associated with type 2 diabetes mellitus//Scientific bulletin, series: Medicine, Pharmacology. - 2014. 3№18(189). - Issue 27. - pp. 50-52; 
[7] Tikhonova P. P., Sokolova L. A. Features of the regulatory mechanisms of the autonomous nervous system in patients with arterial hypertension with violations of the daily profile of arterial pressure (Non dipper type). - 1993. - "Bulletin of Arrhythmology». Published by VA-No. 40 of 15/10. - 2005. - pp. 5054;

[8] Konradi A. O. Interaction between the sympathetic nervous system and pressure //Arterial hypertension. - 2012. - volume 18, No. 6. - pp. 577-583;

[9] Khatami M., Heidari M.M., Soheilyfar S. Common rs5918 (P1A1/A2) polymorphism in the ITGB3 gene and risk of coronary artery disease// Archives of Medical Science Atherosclerotic Diseases.- 2016; 1(1): pp.9-15.

[10] Tuguz A. R., Agadzhanyan N. A., Lysenko S. P., Muzhenya D. V., Ozheva R. Sh., Ashkanova T. M., Anokhina E. N. Frequencies of Met235Thr, Thr174Met polymorphisms of the angiotensinogen gene (AGT) and A1166C alleles of the angiotensinogen - 2 type 1 receptor (AGT2R1) in ethnic groups of the population of Maykop. // Modern problems of science and education-2011. - No. 3-p. 15.

[11] O. S. Glotov, A. S. Glotov, O. A. Tarasenko, T. E. Ivashchenko, V. S. Baranov / Investigation of functionally significant polymorphism of ACE, AGTR1, eNOS, MTHFR, MTRR, and APOE genes in the population of the Northwestern region of Russia// Ecological human genetics. 2004-vol. 11. - No. 3. - pp. 32-35;

[12] Goncharova I. A., Babushkina N. P., Minaicheva L. I., Markova V. V., Kulish E. V.,

Salakhov R. R., Makeeva O. A., Puzyrev V. P. Prevalence of alleles of polymorphic variants of Leu33Pro and Leu66Arg of the ITGB3 gene in residents of the Siberian region // Genetics. - 2013-vol. 49, No. 8. p.p. $1008-1013$

[13] G. V. Dzyak, N. G. Horovenko, T. V. Kolesnik, S. V. Podolskaya, T. A. Andrushchenko The role of angiotensin-converting enzyme gene polymorphism in the realization of the influence of the daily blood pressure profile on the formation of left ventricular hypertrophy in patients with arterial hypertension //Ukrainian Journal of Cardiology. - 2007. - No. 6. pp. 31-39;

\section{Author's information}

Zotova T.Yu. - MD, professor of the V. A. Frolov Department of General Pathology and Pathophysiology, Medical Institute, Peoples' Friendship University of Russia, Moscow, Russia, tel.+7-9636376524, e-mail: zotovat@mail.ru.
Azova M.M.- Doctor of Biological 636376524 Institute, Peoples' Friendship University of Russia, Moscow, Russia, tel.+7-926-236-30-79, e-mail: azovam@mail.ru

Lukanina A.A.- Therapeutist, doctor of Functional Diagnotics, postgraduate student of the V. A. Frolov Department of General Pathology and Pathophysiology, Medical Institute, Peoples' Friendship University of Russia, Moscow, Russia, tel.+7-906-042-64-72, email: lukaninmark@gmail.com

Ait Aissa A. - PhD in Biology, assistant Lecturer of the Department of Biology and General Genetics, Medical Institute, Peoples' Friendship University of Russia, Moscow, Russia, tel.+7-926-423-22-75, e-mail: ait.mira90@hotmail.fr

Blagonravov M.L - MD, professor, Head of the V. A. Frolov Department of General Pathology and Pathophysiology, Medical Institute, Peoples' Friendship University of Russia, Moscow, Russia, tel.+ 7903-194-83-64, e-mail: blagonravovm@mail.ru

For correspondence: Tatjana Zotova - MD, professor of the V. A. Frolov Department of General Pathology and Pathophysiology, Medical Institute, Peoples' Friendship University of Russia, Moscow, Russia, tel.+7-9636376524, e-mail: zotovat@mail.ru.

\section{Author's Contributions.}

\section{All authors contributed to this study:}

Zotova T. Yu - conceptualization, methodology, data curation, writing - original draft preparation, supervision;

Azova M.M - methodology. data curation, visualization, investigation;

Lukanina A.A.- formal analysis, software, investigation, writing - original draft preparation;

Ait Aissa A. - formal analysis, investigation;

Blagonravov M.L - validation, data curation, writing - review and editing, supervision, project administration, funding acquisition.

All authors have read and agreed to the published version of the manuscript.

Funding: No funding.

Institutional Review Board Statement: The study was conducted according to the guidelines of the Declaration of Helsinki, and approved by the Ethics Committee of RUDN University Institute of Medicine (protocol code 10 and date of approval: 20.06.2019).

Informed Consent Statement: Informed consent was obtained from all subjects involved in the study.

Data Availability Statement: Data is contained within the article.

Conflicts of Interest: The authors declare no conflict of interest. 


\section{Creative Commons Attribution License 4.0} (Attribution 4.0 International, CC BY 4.0)

This article is published under the terms of the Creative Commons Attribution License 4.0

https://creativecommons.org/licenses/by/4.0/deed.en_US 